\title{
A novel method for the synthesis of calcium carbonate (aragonite) nanoparticles from cockle shells
}

\begin{abstract}
We report a simple, novel and low-cost method for the synthesis of aragonite nanoparticles from cockle shells. Aragonite is one of the least abundant biogenic polymorphs of calcium carbonate and is widely used as a biomaterial for the repair of fractured bone, development of advanced drug delivery systems, and tissue scaffolds. The method involves a simple mechanical grinding of the micron-sized cockle shell powders in the presence of a non-toxic and non-hazardous biomineralization catalyst, dodecyl dimethyl betaine (BS-12). The method produces rod-shaped aragonite particles with a diameter of $20 \pm 5 \mathrm{~nm}$ with good reproducibility and without any additional impurities. This was confirmed by a combined analysis of variable pressure scanning electron microscopy (VPSEM), transmission electron microscopy (TEM), Fourier transmission infrared spectroscopy (FTIR), thermogravimetric analyzer (TGA), X-ray diffraction spectroscopy (XRD) and energy dispersive X-ray analyser (EDX). The method should find potential applications in industry for the large scale synthesis of aragonite nanoparticles from a low cost but abundant natural resource such as cockle shells.
\end{abstract}

Keyword: Cockle shells; Aragonite nanoparticles; Biomaterials; Biomineralization catalyst 